\title{
Covid-19 and acute kidney injury in hospital: summary of NICE guidelines
}

\author{
Nicholas M Selby professor of nephrology ${ }^{1}$, Lui G Forni professor of intensive care medicine ${ }^{2}$, \\ Christopher M Laing consultant nephrologist ${ }^{3}$, Kerry L Horne specialist trainee in renal medicine ${ }^{4}$, \\ Rhys DR Evans specialist trainee in renal medicine ${ }^{3}$, Bethany $\mathrm{J}$ Lucas NIHR academic clinical fellow \\ in renal medicine ${ }^{1}$, Richard J Fluck consultant nephrologist ${ }^{4}$
}

${ }^{1}$ Centre for Kidney Research and Innovation, University of Nottingham, UK; ${ }^{2}$ Department of Intensive Care, Royal Surrey County Hospital NHS Foundation Trust and Section of Clinical Medicine, School of Biosciences and Medicine, University of Surrey, Guildford, UK; ${ }^{3}$ Department of Renal Medicine, University College London, Royal Free Hospital, London, UK; ${ }^{4}$ Department of Renal Medicine, Royal Derby Hospital, Derby, UK

What you need to know

Acute kidney injury (AKI) may be common in patients with covid-19 and is associated with an increased risk of dying

- AKI associated with covid-19 may be caused by volume depletion, multi-organ failure, viral infection leading directly to kidney tubular injury, thrombotic vascular processes, glomerulonephritis, or rhabdomyolysis

- Maintaining optimal fluid status (euvolaemia) is critical in reducing the incidence of AKI

- Regular assessments of fluid status and fluid management plans are necessary, and in those who need intravenous fluids the choice of replacement fluid should be based on patients' biochemistry and fluid status

- An increased risk of coagulopathy may cause problems with clotting of the extracorporeal circuit during renal replacement therapy

Acute kidney injury (AKI), a sudden reduction in kidney function, is seen in some people with covid-19 infection. A subset of patients develop severe AKI and require renal replacement therapy (RRT). As in many settings, the development of AKI is associated with an increased risk of mortality. ${ }^{12}$ Although our understanding is incomplete, a picture is emerging from case reports and autopsy series of covid-19 specific causes of AKI. Intrinsic renal pathology including thrombotic vascular processes, viral mediated tubular cell injury, and glomerulonephritis have been reported, as well as AKI resulting from extrinsic factors such as fluid depletion, multi-organ failure, and rhabdomyolysis. ${ }^{3-7}$ Anecdotal reports have emerged of proximal tubular injury with Fanconi syndrome that manifests as hypokalaemia, hypophosphataemia, normal anion gap metabolic acidosis, and hypovolaemia from salt wasting. Importantly, AKI can occur at all stages of covid-19 infection, so clinical vigilance and consideration of risk factors for AKI alongside early detection and diagnosis are essential components of general supportive care. Fluid management is central to this.

This article summarises key points from the National Institute for Health and Care Excellence (NICE) covid-19 rapid guideline on AKI in hospital. ${ }^{8}$

\section{Recommendations}

\section{Communicating with patients}

- Communicate effectively with patients, their families, and carers, and support their mental wellbeing to help alleviate any anxiety they may have about covid-19. Signpost to charities and UK government guidance on the mental health and wellbeing aspects of covid-19.

\section{Minimising risk for patients and healthcare workers}

- All healthcare workers involved in receiving, assessing, and caring for patients who have known or suspected covid-19 should follow UK government guidance for infection prevention and control. ${ }^{10}$

- If covid-19 is later diagnosed in a patient not isolated from admission or presentation, follow UK government guidance on management of exposed healthcare workers and patients in hospital settings. ${ }^{11}$

\section{Planning treatment and care}

- Discuss the risks, benefits, and likely outcomes of treatment options with patients with covid-19, and their families and carers. This will help them make informed decisions about 
their treatment goals and wishes, including treatment escalation plans where appropriate.

- Find out if patients have advance care plans or advance decisions to refuse treatment, including "do not attempt cardiopulmonary resuscitation" decisions, and take account of these in planning care.

- Monitor patients for the development or progression of chronic kidney disease (CKD) after AKI. Guidance on care after hospital discharge produced jointly by Think Kidneys and the Royal College of General Practitioners is designed to support safer transitions of care and post-discharge monitoring, and is of relevance to both hospital and general practice teams. ${ }^{12}$

\section{Assessing for AKI in patients with suspected or confirmed covid-19}

Be aware that, in patients with covid-19, AKI

- may be common, but prevalence is uncertain and depends on clinical setting; the Intensive Care National Audit and Research Centre's report on covid-19 in critical care reported that $31 \%$ of patients on ventilators and $4 \%$ not on ventilators needed renal replacement therapy for $\mathrm{AKI}^{13}$

- is associated with an increased risk of dying ${ }^{12}$

- can develop at any time before or during hospital admission

- causes may include volume depletion (hypovolaemia), haemodynamic changes, viral infection leading directly to kidney tubular injury, thrombotic vascular processes, glomerular pathology, or rhabdomyolysis ${ }^{14}$

- may be associated with haematuria, proteinuria, and abnormal serum electrolyte levels (both increased and decreased serum sodium and potassium). ${ }^{1}$

Be aware that in patients with covid-19

- maintaining optimal fluid status (euvolaemia) is critical in reducing the incidence of $\mathrm{AKI}$, but this can be hard to achieve

- treatments being used to manage covid-19 may increase the risk of AKI-for example, diuretics if they have caused volume depletion (hypovolaemia)

- fever and increased respiratory rate increase insensible fluid loss

- dehydration (often needing correction with intravenous fluids) is common on admission to hospital and may also develop later

- risk of coagulopathy is increased.

On hospital admission or transfer, assess for AKI in all patients. Record

- medical history and comorbidities, including factors that further increase the risk of AKI (such as CKD, heart failure, liver disease, diabetes, history of AKI, age 65 or over)

- fluid status by clinical examination (for example, peripheral perfusion, capillary refill, pulse rate, blood pressure, postural hypotension, jugular venous pressure, or pulmonary or peripheral oedema)

- fluid status by fluid balance (fluid intake, urine output, and weight)

- full blood count

- serum urea, creatinine, and electrolytes (sodium, potassium, bicarbonate).
Review the use of medicines that can cause or worsen AKI and stop these unless essential.

- Ask a pharmacist for advice about optimising the choice and dosage of medicines, including anticoagulants for treatment or prophylaxis. More detailed information is available in the Think Kidneys guidelines for medicines optimisation in patients with AKI. ${ }^{15}$

Continue to assess for AKI. Record and monitor fluid status by clinical examination and fluid balance daily. Measure serum urea, creatinine, and electrolytes (sodium, potassium, bicarbonate) at least every 48 hours or more often if clinically indicated (eg, in those at increased risk of AKI, in those who have sustained AKI, and those with electrolyte abnormalities).

Use an early warning score for patients whose clinical condition is deteriorating or who have suspected sepsis:

- NEWS2 has been endorsed by NHS England.

- When using NEWS2 be aware of the Royal College of Physicians' warning that any increase in oxygen requirements should be escalated for clinical review and increased observations. ${ }^{16}$

\section{Detecting and investigating $\mathrm{AKI}$ in patients with suspected or confirmed covid-19}

Detect AKI using NHS England's AKI algorithm ${ }^{17}$ or any of the following criteria:

- an increase in serum creatinine of $\geq 26 \mu \mathrm{mol} / \mathrm{L}$ in 48 hours

- an increase of $\geq 50 \%$ in serum creatinine, known or presumed to have occurred in the past seven days

- a fall in urine output to $\leq 0.5 \mathrm{~mL} / \mathrm{kg} / \mathrm{hour}$ for more than six hours.

Do urinalysis for blood, protein, and glucose to help identify the cause of AKI. Record the results and take action if these are abnormal (including referral if needed; see section below on referral in patients with suspected or confirmed covid-19).

Perform imaging if urinary tract obstruction is suspected.

\section{Managing fluid status in patients with suspected or confirmed covid-19}

- Aim to achieve and maintain optimal fluid status (euvolaemia) in all patients.

- If there is volume depletion (hypovolaemia) and fluid needs cannot be met orally or enterally, give patients intravenous fluids as part of a protocol to restore and maintain optimal fluid status (euvolaemia).

- Ensure patients have an intravenous fluid management plan that is reviewed daily.

- Base choice of fluids on biochemistry results and fluid status. The composition of commonly used fluids is summarised in table 1.

- Do not routinely offer loop diuretics to treat AKI but consider them for treating fluid overload.

\section{Managing hyperkalaemia in patients with suspected or confirmed covid-19}

- Be aware of the risk of hyperkalaemia and manage according to local protocols.

- The potassium binders patiromer and sodium zirconium cyclosilicate can be used alongside standard care for the emergency management of acute life threatening 
hyperkalaemia (these agents have been approved by NICE for this indication)..$^{1920}$

\section{Referral in patients with suspected or confirmed covid-19}

Refer patients with AKI for further specialist advice if

- there is diagnostic uncertainty about the cause of AKI, which may need further tests or imaging

- they have abnormal urinalysis results, which may be a sign of covid-19 induced kidney damage or other intrinsic renal disease

- fluid management needs are complex

- AKI is worsening despite initial management or has not resolved after 48 hours

- the patient has usual indications for renal replacement therapy, particularly if there is no urine output, such as - life threatening hyperkalaemia

- refractory fluid overload

- severe metabolic acidosis.

\section{Renal replacement therapy in patients with suspected or confirmed covid-19}

The scope of the guideline did not include a detailed review of the technical aspects of provision of renal replacement therapy (RRT) in covid-19. Resources were signposted as follows:

- NHS England has produced a clinical guide on renal replacement therapy options in critical care during the coronavirus pandemic for options for patients with usual indications for RRT based on local availability, equipment, supplies, staffing, and local expertise. ${ }^{21}$

- The Renal Association has collated a set of covid-19 resources, which include protocols for RRT. ${ }^{22}$

- Be aware of the anecdotal reports of RRT circuit clotting because of the increased risk of coagulopathy in patients with covid-19.

- No evidence was found on how best to provide anticoagulation during RRT in patients with covid-19.

\section{Areas of uncertainty}

Information regarding renal involvement in covid-19 is extremely limited in several areas, and further evidence is required. Some of the most pressing questions include

- What is the incidence of AKI in hospitalised patients with covid-19, both in and outside of the intensive care unit?

- What, if any, are the typical clinical, laboratory, and urinary features that characterise AKI in the setting of covid-19?

- What are the different histological patterns of renal involvement in covid-19 and how do these relate to clinical presentation?

- What are the long term effects of covid-19 on renal function, including the proportion of survivors who require ongoing renal replacement therapy resulting from end stage kidney disease?

\section{Guidelines into practice}

- Can you identify patients with covid-19 who are at particular risk of sustaining AKI?

- Do you know which patients with covid-19 associated AKI should be referred for specialist advice, and do you know your local referral pathway?

- How should patients who have sustained covid-19 associated AKI be followed up in primary care, and do you know where to find RCGP guidance on AKI care after hospital discharge?

\section{Useful links}

NICE has produced a guideline on AKI: prevention, detection, and management_https://www.nice.org.uk/guidance/ng148)

- For help with fluid management, see the algorithms and composition of commonly used crystalloids in the NICE guideline on intravenous fluid therapy in adults in hospital (https://www.nice.org.uk/guidance/ cg174/resources)

- See Think Kidneys guidelines for medicines optimisation in patients with AKI (https://www.thinkkidneys.nhs.uk/aki/wp-content/uploads/sites/ 2/2016/03/Guidelines-for-Medicines-optimisation-in-patients-with-AKIfinal.pdf)

\section{How this guideline summary was prepared}

The guideline is part of a series of rapid guidelines on coronavirus (covid-19), developed using interim methods. ${ }^{23}$ The guidelines are based on evidence available when the guideline was published on 6 May 2020 plus expert opinion, and have been verified as far as possible by NICE. Recommendations will be reviewed and updated as the knowledge base and expert experience develops. Readers should refer to the full guideline on the NICE website for the latest version of the guidance (https://www.nice.org.uk/guidance/ng175) and see the NICE coronavirus page (https://www.nice.org.uk/covid-19) for additiona NICE rapid guidelines on covid-19.

\section{How patients were involved in the creation of this article}

Patients were not involved in the creation of this article.

Competing interests: NICE has obtained disclosures of interests from all authors.

Provenance and peer review: commissioned; not externally peer reviewed

Cheng $\mathrm{Y}$, Luo R, Wang K, etal . Kidney disease is associated with in-hospital death of patients with COVID-19. Kidney Int 2020;97:829-38. 10.1016/.kint.2020.03.005 32247631

2 Pei G, Zhang Z, Peng J, etal . Renal involvement and early prognosis in patients with COVID-19 pneumonia. J Am Soc Nephrol 2020;31:2020030276. 10.1681/ASN.2020030276 32345702

3 Larsen CP, Bourne TD, Wilson JD, Saqqa O, Sharshir MA. Collapsing glomerulopathy in a patient with coronavirus disease 2019 (COVID-19). Kidney Int Rep 2020. 10.1016/.ekir.2020.04.002 32292867

4 Nasr SH, Kopp JB. COVID-19-associated collapsing glomerulopathy: an emerging entity. Kidney Int Rep 2020. . 10.1016/j.ekir.2020.04.030 32368701

5 Peleg Y, Kudose S, D'Agati V, etal . Acute kidney injury due to collapsing glomerulopathy following COVID-19 infection. Kidney Int Rep 2020 . 10.1016/..ekir.2020.04.01732346659

6 Su H, Yang M, Wan C, etal. Renal histopathological analysis of 26 postmortem findings of patients with COVID-19 in China. Kidney Int 2020; S0085-2538(20)30369-0. 10.1016/j.kint.2020.04.003 32327202

7 Gross O, Moerer O, Weber M, Huber TB, Scheithauer S. COVID-19-associated nephritis: early warning for disease severity and complications? Lancet 2020; 10.1016/S0140-6736(20)31041-2

8 National Institute for Health and Care Excellence. COVID-19 rapid guideline: acute kidney injury in hospital (NG175): NICE. 2020. https://www.nice.org.uk/guidance/NG175.

9 Public Health England. Guidance for the public on the mental health and wellbeing aspects of coronavirus (COVID-19). 2020. https://www.gov.uk/government/publications/covid-19guidance-for-the-public-on-mental-health-and-wellbeing/guidance-for-the-public-on-themental-health-and-wellbeing-aspects-of-coronavirus-covid- 19.

10 Public Health England COVID-19: infection prevention and control (IPC). 2020. https:// www.gov.uk/government/publications/wuhan-novel-coronavirus-infection-prevention-andcontrol.

11 Public Health England. COVID-19: management of exposed staff and patients in health and social care settings. 2020. https://www.gov.uk/government/publications/covid-19management-of-exposed-healthcare-workers-and-patients-in-hospital-settings

12 Royal College of General Practitioners, Think Kidneys AKI Programme. Guidance on the timeliness of post-discharge care for adults following acute kidney injury. 2020. https:// www.rcgp.org.uk/clinical-and-research/resources/a-to-z-clinical-resources/acute-kidneyinjury.aspx

13 Intensive Care National Audit and Research Centre. ICNARC report on COVID-19 in critical care. Case Mix Programme Database, 2020.

14 Batlle D, Soler MJ, Sparks MA, et al. Acute kidney injury in COVID-19: emerging evidence of a distinct pathophysiology. J Am Soc Nephrol 2020:ASN.2020040419.

15 Think Kidneys. Guidelines for medicines optimisation in acute kidney injury 2016. 2016. https://www.thinkkidneys.nhs.uk/aki/wp-content/uploads/sites/2/2015/06/Medicinesoptimisation-toolkit-for-AKI-FINAL.pdf.

16 Royal College of Physicians. NEWS2 and deterioration in COVID-19. 2020. https://www. rcplondon.ac.uk/news/news2-and-deterioration-covid-19

17 NHS England. Acute kidney injury (AKI) algorithm. 2020. https://www.england.nhs.uk akiprogramme/aki-algorithm/

18 National Institute for Health and Care Excellence. Intravenous fluid therapy in adults in hospital (NICE Guideline CG174): NICE. 2020. https://www.nice.org.uk/guidance/cg174.

19 National Institute for Health and Care Excellence. Patiromer for treating hyperkalaemia. Technology appraisal guidance [TA623]. 2020. https://www.nice.org.uk/guidance/ta623

20 National Institute for Health and Care Excellence. Sodium zirconium cyclosilicate for treating hyperkalaemia. Technology appraisal guidance [TA599] 2019. https://www.nice. org.uk/guidance/ta599 
21 NHS England. Clinical guide for renal replacement options in critical care during the COVID-19 pandemic. 2020. https://www.england.nhs.uk/coronavirus/wp-content/uploads/ sites/52/2020/04/C0298-speciality-guide-clinical-guide-for-renal-replacement-therapyoptions-in-critical-care-v1.1.pdf

22 The Renal Association. COVID-19: Information and guidance for renal professionals: @renalassoc; 2020. https://renal.org/covid-19/
23 National Institute for Health and Care Excellence. Interim process and methods for developing rapid guidelines on COVID-19: NICE. 2020. https://www.nice.org.uk/process/ pmg35/chapter/scoping

Published by the BMJ Publishing Group Limited. For permission to use (where not already granted under a licence) please go to http://group.bmj.com/group/rights-licensing/ permissions 


\section{Table}

Table 1 | Composition of commonly used fluids, adapted from NICE Guideline (CG174) ${ }^{18}$

\begin{tabular}{|c|c|c|c|c|c|c|c|c|c|c|}
\hline Content & Plasma & $\begin{array}{c}\text { Sodium } \\
\text { chloride } \\
0.9 \%\end{array}$ & $\begin{array}{c}\text { Sodium } \\
\text { chloride } \\
0.18 \% / 4 \% \\
\text { glucose }\end{array}$ & $\begin{array}{c}\text { Sodium } \\
\text { chloride } \\
0.45 \% / 4 \% \\
\text { glucose }\end{array}$ & $\begin{array}{c}5 \% \\
\text { glucose }\end{array}$ & Hartmann's & $\begin{array}{l}\text { Lactated } \\
\text { Ringer's }\end{array}$ & $\begin{array}{l}\text { Ringer's } \\
\text { acetate }\end{array}$ & $\begin{array}{l}\text { Alternative } \\
\text { balanced } \\
\text { solutions for } \\
\text { resuscitation }{ }^{\star *}\end{array}$ & $\begin{array}{c}\text { Alternative } \\
\text { balanced } \\
\text { solutions for } \\
\text { maintenance }\end{array}$ \\
\hline $\mathrm{Na}^{+}(\mathrm{mmol} / \mathrm{L})$ & $135-145$ & 154 & 31 & 77 & 0 & 131 & 130 & 130 & 140 & 40 \\
\hline $\mathrm{Cl}^{-}(\mathrm{mmol} / \mathrm{L})$ & $95-105$ & 154 & 31 & 77 & 0 & 111 & 109 & 112 & 98 & 40 \\
\hline $\mathrm{Na}^{+}: \mathrm{Cl}^{-}$ratio & $1.28-1.45: 1$ & $1: 1$ & $1: 1$ & $1: 1$ & - & $1.18: 1$ & 1.19:1 & $1.16: 1$ & $1.43: 1$ & $1: 1$ \\
\hline $\mathrm{K}^{+}(\mathrm{mmol} / \mathrm{L})$ & $3.5-5.3$ & * & * & * & * & 5 & 4 & 5 & 5 & 13 \\
\hline $\mathrm{HCO}^{-}(\mathrm{mmol} / \mathrm{L})$ & $24-32$ & 0 & 0 & 0 & 0 & $\begin{array}{c}29 \\
\text { (lactate) }\end{array}$ & 28 (lactate) & 27 (acetate) & $\begin{array}{c}27 \text { (acetate) } \\
23 \text { (gluconate) }\end{array}$ & $\begin{array}{c}16 \\
\text { (acetate) }\end{array}$ \\
\hline $\mathrm{Ca}^{2+}(\mathrm{mmol} / \mathrm{L})$ & $2.2-2.6$ & 0 & 0 & 0 & 0 & 2 & 1.4 & 1 & 0 & 0 \\
\hline $\mathrm{Mg}^{2+}(\mathrm{mmol} / \mathrm{L})$ & $0.8-1.2$ & 0 & 0 & 0 & 0 & 0 & 0 & 1 & 1.5 & 1.5 \\
\hline $\begin{array}{l}\text { Glucose } \\
(\mathrm{mmol} / \mathrm{L})\end{array}$ & $3.5-5.5$ & 0 & 222 & 222 & 278 & 0 & 0 & 0 & 0 & 222 \\
\hline $\mathrm{pH}$ & $7.35-7.45$ & $4.5-7$ & 4.5 & & $3.5-5.5$ & $5-7$ & $6-7.5$ & $6-8$ & $4-8$ & $4.5-7$ \\
\hline $\begin{array}{l}\text { Osmolarity } \\
\text { (mOsm/L) }\end{array}$ & $275-295$ & 308 & 284 & & 278 & 278 & 273 & 276 & 295 & 389 \\
\hline
\end{tabular}

* These solutions are available with no potassium or differing quantities of potassium already added

** Alternative balanced solutions are available commercially under different brand names and composition may vary by preparation 